\title{
The clinicopathological study of lung cancer concealed in end-stage of interstitial lung disease
}

\author{
Bei Wang ${ }^{1}$, Xiaoyan Zhang ${ }^{2,3,4}$, Huang Chen ${ }^{1}$, Lei Yang ${ }^{1}$, Jie Li ${ }^{1}$, Fei Xiao ${ }^{5}$, Chaoyang Liang ${ }^{5}$, \\ Dingrong Zhong ${ }^{1}$
}

${ }^{1}$ Department of Pathology, ${ }^{2}$ Department of Pulmonary and Critical Care Medicine, China-Japan Friendship Hospital, Beijing 100029, China; ${ }^{3}$ National Clinical Research Center for Respiratory Diseases, Beijing 100191, China; ${ }^{4}$ Institute of Respiratory Medicine, Chinese Academy of Medical Sciences, Beijing 100191, China; ${ }^{5}$ Department of Thoracic Surgery, China-Japan Friendship Hospital, Beijing 100029, China

Contributions: (I) Conception and design: B Wang; (II) Administrative support: D Zhong; (III) Provision of study materials or patients: X Zhang, F Xiao, C Liang; (IV) Collection and assembly of data: H Chen, J Li, L Yang; (V) Data analysis and interpretation: B Wang, X Zhang; (VI) Manuscript writing: All authors; (VII) Final approval of manuscript: All authors.

Correspondence to: Dingrong Zhong. Department of Pathology, China-Japan Friendship Hospital, Yinghuayuan Dongjie No. 2, Beijing 100029, China. Email: txzzzryy@163.com.

\begin{abstract}
Background: Most of the patients with interstitial lung disease (ILD) complicated with lung cancer (ILDLC) showed non-specific clinical manifestations. This study is to explore the incidence of lung cancer concealed in the end-stage of interstitial lung disease (LC-CES-ILD).

Methods: A total of 154 cases of lung transplantation from March 2017 to December 2018 were studied retrospectively, of which 7 cases were found to be LC-CES-ILD. Serum tumor biomarkers were examined. $\mathrm{HE}$ and immunohistochemical staining were performed for the tumor tissue after the operation. Lung cancer (LC) drive gene was detected by quantitative real-time polymerase chain reaction (qRT-PCR).
\end{abstract}

Results: The percentage of male patients with idiopathic pulmonary fibrosis (IPF) was $44.81 \%$. The expression of all tumor biomarkers was significantly increased in 6 patients with LC-CES-ILD. After operation, apparent destruction of lung tissue structure was observed in 7 patients, and honeycomb appearance could be seen in some areas. After HE staining, 4 cases of acinar type and 2 cases of mucinous adenocarcinoma were found. The results of molecular pathology showed that only one case of mucinous adenocarcinoma had KRAS mutation, and no mutation of LC co-driving gene was found in the rest of the cases.

Conclusions: It is necessary to detect the lung tissue of patients with end-stage ILD, which were probably correlated with the occurrence of LC concealed, before lung transplantation.

Keywords: Interstitial lung disease (ILD); lung cancer (LC); lung cancer concealed in end-stage of interstitial lung disease (LC-CES-ILD); lung transplantation

Submitted Aug 02, 2019. Accepted for publication Nov 08, 2019.

doi: $10.21037 /$ tcr.2019.11.36

View this article at: http://dx.doi.org/10.21037/tcr.2019.11.36

\section{Introduction}

Interstitial lung disease (ILD) is also known as diffuse parenchymal lung disease (DPLD). It is a kind of lung disease that involves alveolar wall and alveolar cavity and has different grade of inflammation and fibrosis. At present, the general respiratory disease community adopts the classification of ILD and idiopathic ILD recommended by the American Thoracic Society (ATS) and the European Respiratory Disease Society (ERS) (1).

The pathological characteristics of patients with lung cancer (LC) are malignant proliferation of tumor cells, but the etiology of ILD is only a few known. However, no matter what the cause of ILD, lung fibroblasts will show abnormal or malignant proliferation, which is similar to the 
pathological characteristics of LC patients.

When ILD develops to the end stage, lung transplantation becomes an effective method for the treatment of end-stage lung disease (2). While, the incidence of LC in ILD patients is increasing. Hitherto, the relationship between ILD and the pathogenesis of LC is not clear. Because most of the clinical manifestations of ILD complicated with LC (ILD-LC) are non-specific, when ILD-LC is diagnosed, it often progresses to the advanced stage of LC. Consequently, how to diagnose the disease in the early stage of ILD-LC has become a serious problem in clinical practice.

With the progress of medical technology, the strategy of diagnosis and treatment of LC has changed from cellular level to precise medical treatment based on molecular level (3). Drive genes are essential genes related to tumorigenesis and development, which play a crucial role in the induction and proliferation of LC. Epidermal growth factor receptor (EGFR) gene, kirsten rat sarcoma viral oncogene (KRAS) gene, B-type Raf kinase (BRAF) gene, human epidermal growth factor receptor-2 (Her-2) gene, and other genes are the most commonly used LC drive genes. It is beneficial to develop the best possible treatment option LC by detecting the driving genes in these ILD-LC.

After routine diagnostic procedures, the cases of LC do not occur in the lungs removed from lung transplants. However, 154 cases of lung transplantation were completed in Sino-Japanese Friendship Hospital within two years, of which 7 cases were not found or could not be diagnosed before operation. However, in the lung transplanted out of the lung, pathological examination found that it was finally diagnosed as terminal qualitative disease complicated with LC. This kind of case can be called lung cancer concealed in end-stage of interstitial lung disease (LC-CES-ILD). We retrospectively studied these rare cases and discussed the clinicopathological features.

\section{Methods}

\section{Patients}

We reviewed all cases of lung transplantation in ChinaJapan Friendship Hospital from March 2017 to December 2018. According to the criteria of the ATS (4), patients with severe lung diseases diagnosed by imaging or pathology and recommended for lung transplantation (2). A total of 154 patients were recruited. Inclusion criteria: (I) patients who met the criteria of ATS and ERS for lung transplantation;
(II) cardiac function of the New York Heart Association (NYHA) III or IV; (III) the experimental treatment of intravenous administration of vasocyclin or similar equivalent preparation is ineffective; (IV) cardiac index (CI) $<2 \mathrm{~L} /\left(\min \cdot \mathrm{m}^{2}\right)$. Exclusion criteria: (I) the receptor has a history of malignant tumors in the past 2 years, but localized malignant tumors; (II) the receptor has other primary organ dysfunction that can't be treated (such as heart, liver, kidney, and brain); (III) the patient does not agree to a lung transplant, or mental or psychological problems are unable to cooperate with the medical team and do not comply with the doctor's diagnosis and treatment.

These cases, who are not diagnosed with a tumor before the operation, but LC was found in the transplanted lung, was screened from 154 lung transplant cases for inclusion in this study. The clinical data, preoperative imaging data, laboratory examination, and pathological data of these patients were collected.

\section{Preoperative pathological examination}

The fasting venous blood of $3 \mathrm{~mL}$ was taken and the serum was separated for examination. Microparticle enzyme immunoassay (MEIA) was used. The experimental operation was carried out in strict accordance with the relevant guidelines and instructions, and the changes of serum tumor marker carcinoembryonic antigen (CEA), carbohydrate antigen 125 (CA 125), CA-153, neuron-specific enolase (NSE), cytokeratin 19-fragments (CYFRA21-1), CA72-4, CA199 in the samples were analyzed. Positive reference values: CEA $(<5 \mathrm{ng} / \mathrm{mL})$, CA125 $(<35 \mathrm{U} / \mathrm{mL})$, CA-153 $(<25 \mathrm{U} / \mathrm{mL})$, NSE $(<16.3 \mathrm{ng} / \mathrm{mL})$, CYFRA21-1 $(<3.3 \mathrm{ng} / \mathrm{mL})$, CA72-4 (<6.9 U/mL), CA199 ( $<27 \mathrm{U} / \mathrm{mL})$.

\section{Treatment and observation of lung transplantation samples}

The diseased lungs resected after lung transplantation were treated with routine pathological specimens. The specimens were perfused with $10 \%$ neutral formalin and fixed overnight. The pathologist scrutinized the specimen according to the standard of lung tissue sampling. Each lung lobe was taken separately, and the number of samples was determined according to the sample situation. The volume of material was at least three tissue blocks/lung lobes. Thus, the suspicious tumor tissue found in naked eye examination, and the number of tissue blocks was increased. Besides, the severed bronchial end, severed vascular end and lymph nodes in the specimen were routinely taken. 


\section{Hematoxylin-eosin stain (HE) and immunobistochemical detection}

The tissue was embedded in paraffin and cut into sections of $4 \mu \mathrm{m}$ thickness. The sections were stained with $\mathrm{HE}$ and observed under the microscope. Immunohistochemistry was performed by EnVision method. The primary antibodies (TTF-1, p63, Ki67, and p53) were purchased from Beijing Zhongshan Jinqiao Biotechnology Co., Ltd. The second antibody and chromogenic agent were purchased from Roche Diagnostic products (Shanghai) Co., Ltd. The immunohistochemical experiment was carried out according to the reagent instructions, and the corresponding negative and positive controls were established. All pathological sections were read independently by two senior pathologists.

\section{Molecular biological examination}

Ten paraffin specimens of tumor with a thickness of $5 \mu \mathrm{m}$ were selected for each case. Human LC polygene combined detection kit (Eddard Company) was used to extract DNA, and the fluorescence PCR method was used to detect the LC drive gene. The genes tested included EGFR, reactive oxygen species ROS1, BRAF, anaplastic lymphoma kinase (ALK), $K R A S$, receptor tyrosine kinase gene (RET), neuroblastoma RAS viral oncogene bomolog (NRAS), Her-2, $\alpha$-isoform of phosphatidylinositol 3-kinase (PIK3Ca), Methoprene-tolerant (Met). The detailed information of these genes was shown in Table S1.

\section{Statistical analysis}

SPSS 22.0 (SPSS Inc., Chicago, IL, USA) was used for statistical analysis. The proportion of patients is represented by $n \%$.

\section{Results}

\section{Clinical information of 154 patients undergoing lung transplantation}

As shown in Table 1, the male patients accounted for $87 \%$ of the 154 lung transplant patients, and $78.57 \%$ of the patients were 50-69 years old. The primary type of disease was idiopathic pulmonary fibrosis (IPF), which accounted for $44.81 \%$ of the transplanted patients. There was no significant difference in the proportion of bilateral lung sequential transplantation, left single lung transplantation and right single lung transplantation among the three groups.

\section{LC-CES-ILD patient clinical information}

A total of 7 cases of LC were diagnosed pathologically after operation. These ILD patients were not diagnosed as LC before transplantation, but they were found to belong to LC-CES-ILD after transplantation. The clinical information is shown in Table 2.

Before transplantation, the clinical diagnosis suspected the five patients of having tumors, but it was not ensured before the operation. However, only No. 2 and No. 7 patients were confirmed to be LC by pathological examination after the surgery. All seven patients were male, with an average age of 57.57 years (46-64 years) and a history of severe smoking (15-90 package years). The primary diseases of lung transplantation were IPF (4 cases), connective tissue disease-associated interstitial lung disease (CTD-ILD) (2 cases), and chronic obstructive pulmonary disease (COPD) (1 case). Two of the patients underwent bilateral sequential lung transplantation, while the others underwent unilateral lung transplantation.

The interval between the last computed tomography (CT) examination and operation was 2-29 days. None of these patients was diagnosed as tumors by CT. Alternatively, positron emission tomography-CT (PET-CT) screening was performed in with No. 3 and No. 6 patients before operation, but the cancer could not be confirmed. Serum tumor biomarkers were screened in 7 patients before the surgery, and CEA, CA125, CA-153, NSE, CYFRA21-1, CA72-4, CA199 was significantly increased in 6 patients. However, the serum biomarkers of No. 2 patients were only slightly elevated in CA125, and the other serum tumor biomarkers were normal. Therefore, this patient was diagnosed as tumor-free before operation.

\section{Detection of tumor tissue in postoperative LC-CES-ILD patients}

The tumor tissue of 5 patients with LC-CES-ILD involved the upper lobe, while two patients involved two lobes of the lung. The lung tissue structure of 7 patients was destroyed, the texture was medium to tight, and honeycomb appearance could be seen in some areas. In some cases, the presence of mucus is prominent, and the boundary of the surrounding lung tissue is unclear (for example, No. 5 patients, Figure 1A).

Three cases (patient: No. 1, No. 3, No. 4) with tumor symptoms were found by accident during experiential sampling. Moreover, it is still impossible to accurately 
Table 1 The basic clinical information of 154 lung transplant patients

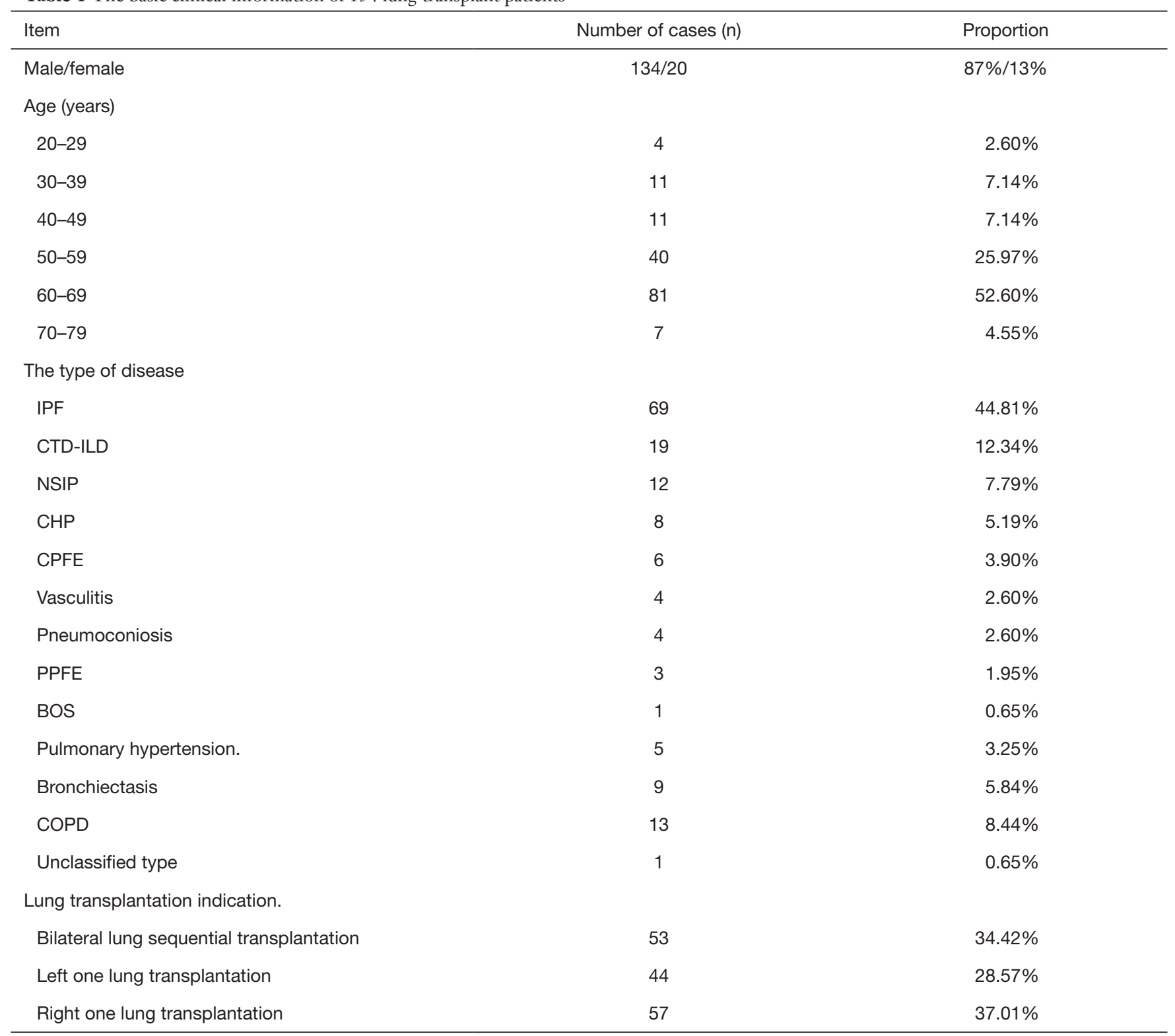

IPF, idiopathic pulmonary fibrosis; CTD-ILD, connective tissue disease-associated interstitial lung disease; NSIP, non-specific interstitial pneumonia; CHP, chronic hypersensitivity pneumonia; CPFE, combined pulmonary fibrosis and emphysema; PPFE, pleuroparenchymal fibroelastosis; COPD, chronic obstructive pulmonary disease; BOS, obliterative bronchiolitis syndrome.

determine the location and size of the tumor with the naked eye at the time of re-examination of the specimen (for example, patient No. 4, Figure 1B). Four cases were single tumor lesions and three cases where two tumor lesions (Table 3).

HE staining showed that the alveolar structure of lung tissue in LC-CES-ILD patients almost disappeared. The interstitial fibrosis was high, and honeycomb lung and fibroblast hyperplasia lesions were formed in some areas of the lung. Multifocal alveolar epithelial hyperplasia, squamous or mucinous epithelialization, and atypical hyperplasia occurred. Infiltration and aggregation of lymphocytes and thickening of pleura could be seen in some areas of the lung. Histologically, most of the patients were lung adenocarcinoma (6/7), 4 cases were acinar type, and 2 cases were mucinous adenocarcinoma (Figure 2). Patients with No. 6 have a small number of pleomorphic cancer components. Microscopically, the tumor 
Table 2 The preoperative clinical information of 7 patients with LC-CES-ILD

\begin{tabular}{|c|c|c|c|c|c|c|c|}
\hline Item & \multicolumn{7}{|c|}{ Case number (No.) } \\
\hline Gender & Male & Male & Male & Male & Male & Male & Male \\
\hline Age & 59 & 46 & 63 & 64 & 52 & 61 & 58 \\
\hline Smoking index (bag/year) & 20 & 90 & 70 & 20 & 30 & 40 & 15 \\
\hline IPF & + & & + & & + & & + \\
\hline COPD & & + & & & & & \\
\hline CTD-ILD & & & & + & & + & \\
\hline \multicolumn{8}{|c|}{ Serum tumor biomarkers detected before operation (nit of marker, range of normal value) } \\
\hline $\mathrm{CA}-153(\mathrm{U} / \mathrm{mL},<25)$ & 193.2 & 6.44 & 34.26 & 221.3 & 58.26 & 74.61 & 38.73 \\
\hline NSE (ng/mL, <16.3) & 18.27 & 13.37 & - & 12.94 & 16.95 & - & - \\
\hline CYFRA21-1 (ng/mL, <3.3) & 7.3 & 2.87 & 4.73 & 6.16 & 6.42 & 3.41 & 5.22 \\
\hline CA72-4 (U/mL, <6.9) & 9.94 & 2.45 & 10.01 & 17.03 & 17.57 & - & - \\
\hline CA199 (U/mL, <27) & - & - & 51.11 & 583.4 & 28.93 & 525.8 & 284.1 \\
\hline \multicolumn{8}{|l|}{ The results of the last CT examination before operation } \\
\hline Interstitial pneumonia & + & + & + & + & & & + \\
\hline Mediastinal lymphadenopathy & + & & & & & & \\
\hline No tumor & & + & & & & & + \\
\hline \multicolumn{8}{|l|}{ Transplantation type } \\
\hline Bilateral lung sequential & & + & & + & & & \\
\hline Right single lung & + & & & & + & & + \\
\hline Left single lung & & & + & & & + & \\
\hline
\end{tabular}

IPF, idiopathic pulmonary fibrosis; CTD-ILD, connective tissue disease-associated interstitial lung disease; COPD, chronic obstructive pulmonary disease.

cells were fusiform, which has obvious heteromorphism and more mitosis. Only the tumor cells in No. 4 patients were keratinizing squamous cell carcinoma (Figure 3).

Therefore, we can find that the boundaries of tissue between LC and ILD is not clear, as the tumor center or edge is often visible with the radical lesions of the base cells, squamous cell carcinoma surrounding the phenomenon of epidermal squamous, mucus adenocarcinoma surrounding visible dermalbiosis phenomenon, which suggests that the occurrence of tumor stoma has a certain relationship with 

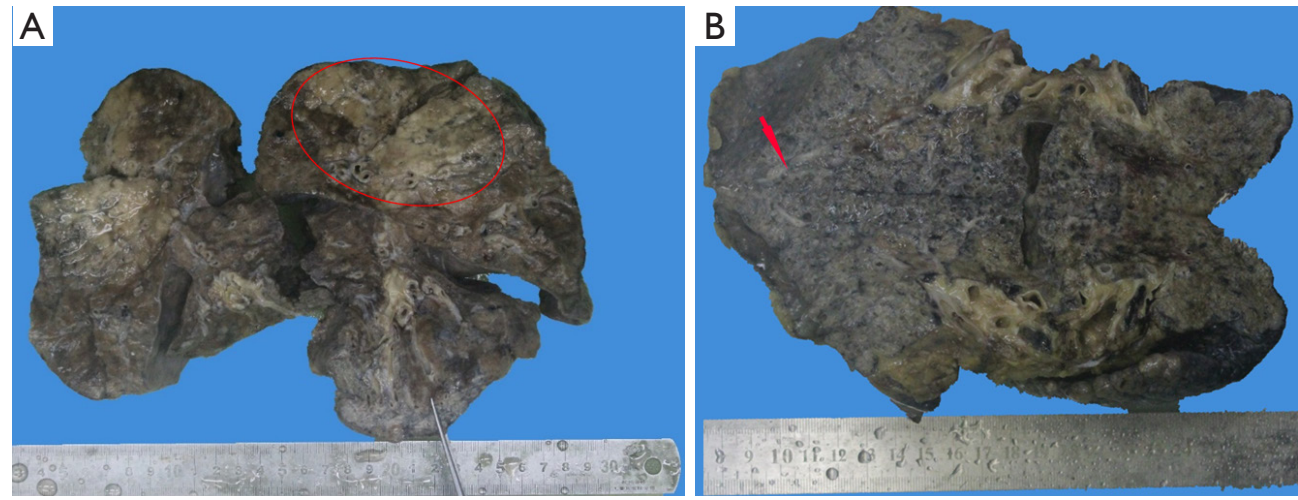

Figure 1 Specimen of the lung lobe of No. 5 patient. (A) Right lobe, visible honeycomb lung changes (red circle); (B) left lobe, most of the lung tissue consolidation (red arrow).

the metastasis and proliferation of ILD.

Three of the patients were involved the visceral pleura, and 3 patients detected lymph node metastasis. Postoperative pathology stages show 1 case in stage Ia 1,3 cases in stage IIb, and 3 cases in stage IIIa. Immunohistochemistry showed that TTF-1 was positive (Figure $4 A$ ) and p63 was negative (Figure $4 B$ ) in adenocarcinoma, a few cells were TTF-1 positive and did not express p63 in mucinous adenocarcinoma, p63 was diffusely positive, and TTF-1 was negative in squamous cell carcinoma. The expression of Ki-67(Figure 4C) was more than $10 \%$ in all tumor tissues and p53 positive (Figure $4 D$ ) in some tumor tissues (Table 3).

The results of the molecular pathological examination showed that only one case of mucinous adenocarcinoma had KRAS mutation, and no mutation of LC drive gene was detected in the rest of the patients (Table S2). Followup indicated that two patients died of infection after transplantation; the rest of the patients were followed up for 68-347 days, and three patients had tumor metastasis (Table 4).

\section{Discussion}

LC and ILD also have many similarities in the pathogenesis, in addition, the two diseases have many similar risk factors, including smoking, advanced age, and so on (5). When ILD occurs, cytokines, chemokines, and growth factors promote the fibrosis process, and then lead to the destruction of the lung tissue structure, thus resulting in a series of reactions, and eventually develop into neoplastic lesions $(6,7)$.

It is also different for the incidence of LC induced by different types of ILD. The incidences of LC were $1.43 \%$ in ILD-only groups (8), however, the incidence of LC was
$4.4 \%$ to $48 \%$ in patients with IPF (9), while the prevalence of LC was as high as $8.9 \%$ in patients with pulmonary fibrosis complicated with emphysema (CPFE) (10). Therefore, it is confirmed that the incidence of ILDLC may be underestimated because it is not detected from the LC-CES-ILD patients in our study, and the actual prevalence of ILD-LC may be higher than the data reported in the literature. Therefore, it is challenging to discover LC based on ILD, which is also a difficult point in the diagnosis and treatment of interstitial diseases.

It has been reported that the probability of accidental detection of LC in transplanted resected lungs is $0.8-2.2 \%$ (11), compared with a slightly higher proportion. Seven patients performed high-resolution CT screening before transplantation. The interval was only 2-29 days, which was significantly lower than the range in reference (12), but still failed to reduce the removal of ILD-LC from the lungs effectively. It is suggested that more effective methods are needed for the preoperative diagnosis of ILD-LC.

It is the key to confirming ILC-CES-LC by careful naked eye observation of the diseased lungs resected in end-stage ILD transplantation. Adenocarcinoma was the most common histological type of ILD-LC, accounting for $43 \%$ (11), followed by squamous cell carcinoma. Most of our cases were adenocarcinoma. The differentiation of mucinous adenocarcinoma was found in several cases, and a rare pleomorphic cancer component was found in one case (12). Hata et al. (13) summarized the surgical specimens and found that ILD-LC had a higher proportion of pleural invasion than LC alone. Three/seven cases in this study also confirmed the destruction of visceral pleura, for the prognostic significance of this phenomenon, more cases are needed to accumulate summary further. 
Table 3 The detection of tumor tissues in 7 patients with LC-CES-ILD

\begin{tabular}{|c|c|c|c|c|c|c|c|}
\hline Item & \multicolumn{7}{|c|}{ Case number (No.) } \\
\hline \multicolumn{8}{|l|}{ Histological types } \\
\hline Invasive adenocarcinoma & + & + & + & & & & + \\
\hline Squamous cell carcinoma & & & & + & & & \\
\hline \multicolumn{8}{|l|}{ Histological subtype } \\
\hline Acinar type & $+80 \%)$ & + & $+(70 \%)$ & & + & $+(60 \%)$ & $+(80 \%)$ \\
\hline Solid type & $+(20 \%)$ & & & & & & $+(20 \%)$ \\
\hline Number of tumor lesions & 1 & 2 & 2 & 1 & 1 & 1 & 2 \\
\hline Tumor size (cm) & 1.5 & $0.8 / 0.15$ & $1.2 / 0.3$ & 0.7 & 7.5 & 10 & $0.8 / 1.5$ \\
\hline Location of tumor in lung & Upper left & Upper left & Upper left & Lower left & $\begin{array}{l}\text { Upper right and } \\
\text { middle right }\end{array}$ & Upper left & Lower right \\
\hline \multicolumn{8}{|c|}{ Identification of tumor by naked eye } \\
\hline Unrecognizable & + & + & + & + & & & \\
\hline Visible & & & & & + & + & + \\
\hline Stage of pTNM & $\mathrm{Ilb}$ & $\mathrm{Ilb}$ & $\mathrm{Ilb}$ & la1 & Illa & Illa & Illa \\
\hline \multicolumn{8}{|l|}{ Immunohistochemical results } \\
\hline TTF-1 & + & + & + & - & + Partial & - & + \\
\hline P63 & - & - & - & + & - & - & - \\
\hline Ki-67 & $+(10 \%)$ & $+(50 \%)$ & $+(20 \%)$ & $+(15 \%)$ & $+(50 \%)$ & $+(20 \%)$ & $+(40 \%)$ \\
\hline P53 & + Partial & + & - & + Partial & - & + Partial & + Partial \\
\hline
\end{tabular}

The pathological diagnosis of LC from ILD, especially invasive tumors, is not difficult, but squamous epithelial metaplasia and hyperplasia of squamous epithelium or mucinous epithelium often occur at the same time of interstitial fibrosis, which is similar to the carcinomatous interstitial reaction of tumor. This needs to be differentiated from invasive tumors. The deletion of $\mathrm{p} 63$ positive basal cells can assist in the diagnosis of invasive adenocarcinoma. The expression of p53 and Ki67 was significantly increased in tumors $(14,15)$, which has a particular significance in differential diagnosis.

Detecting the ILD-LC drive gene, we discovered the mutation of KRAS in only one case of mucinous adenocarcinoma, and the results were consistent with those reported in the literature $(16,17)$. The mutation 

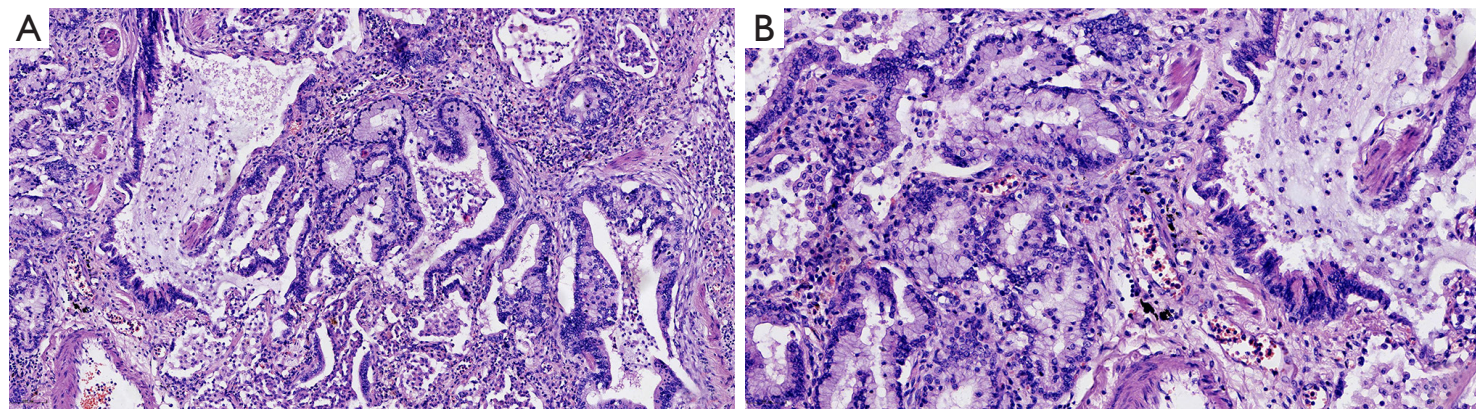

C
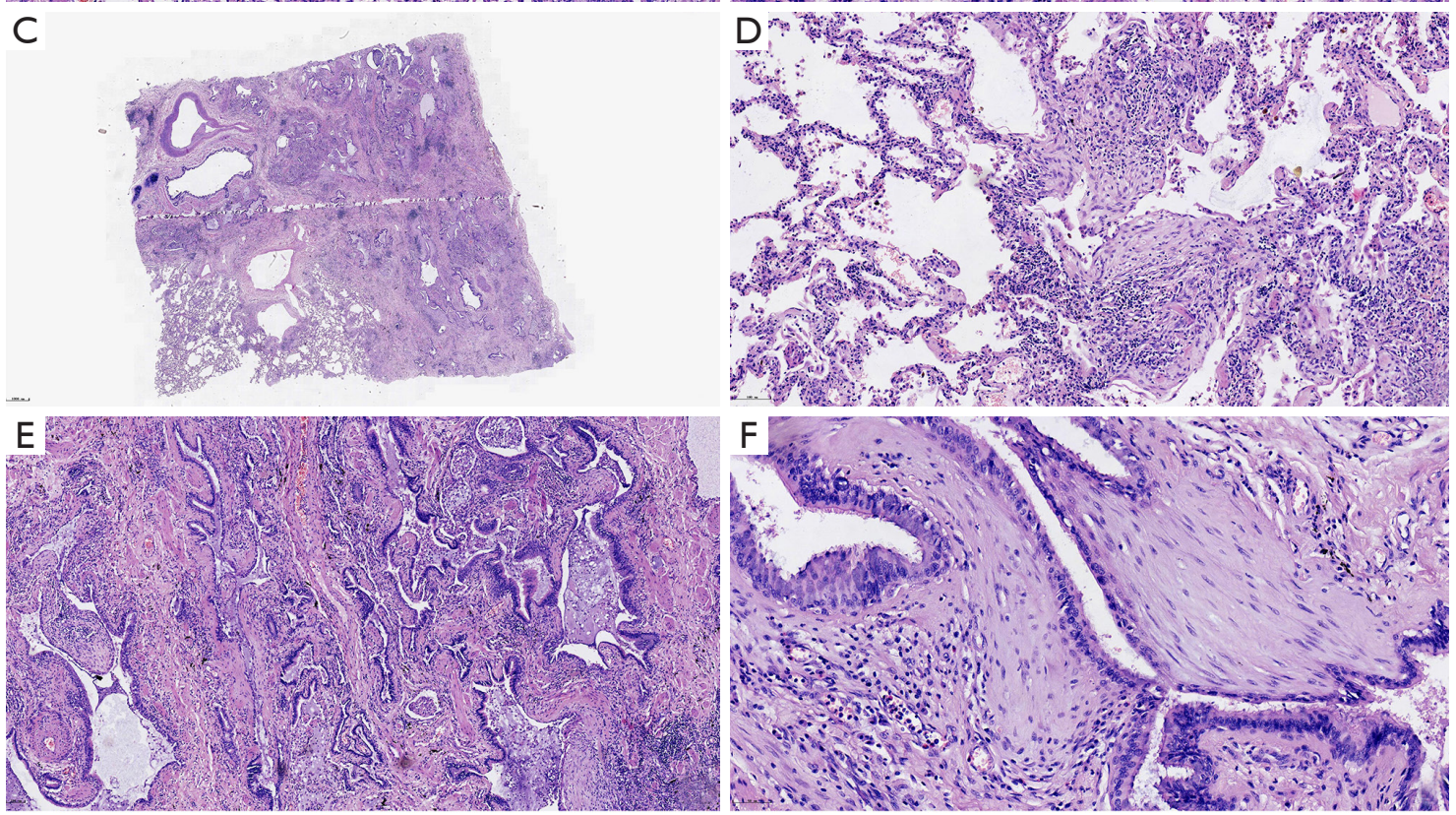

Figure $2 \mathrm{HE}$ of lung tissue in No.5 patient. (A) Abnormal mucous glands were stained with HE in the infiltrating growth area, $\times 20$ times. Interstitial fibrous tissue hyperplasia, local lesions can be seen residual metaplastic mucous epithelium (shown by the blue arrow). (B) HE staining of abnormal mucous glands, $\times 40$ times. The nucleus of the tumor was obviously irregular, the nucleus was located at the bottom of the base, the mucous vesicles could be seen in the cytoplasm, and mucus retention could be seen in the glandular cavity. (C) Ultra-lowpower scanning slice image HE staining. The pathological changes in the surrounding lung tissue were diffusely distributed. Fibrous tissue proliferated, showing patchy distribution, $\times 1$ time. (D) HE staining, $\times 20$ times, in the areas with mild lesions of peripheral interstitial disease. A slightly basophilic focus of fresh fibroblast hyperplasia can be seen. (E) HE staining, $\times 10$ times. The surrounding lung tissue showed fibrosis, the lung structure was obviously destroyed, forming clusters of different sizes of a cystic cavity, and showed honeycomb changes. The wall of the capsule is fibrous tissue, covered by bronchiole epithelium, and contains mucus and inflammatory cells in the cavity. (F) HE staining around the fibrous tissue, $\times 40$ times. The proliferation of fibroblasts and myofibroblasts could be observed. The focus of fibroblastic hyperplasia composed of mucinous blue staining matrix is located around the dense and hyperplastic fibrous tissue.

rate of EGFR in ILD-LC was significantly lower than that in LC patients without ILD, but the mutation of KRAS was relatively common, indicating that ILD-LC may be different from LC without ILD in pathogenesis and molecular phenotype. At the same time, the effect of targeted drugs on ILD-LC is minimal, therefore, the difference between the two should be studied, and the effective treatment for ILD-LC should be studied further.

\section{Conclusions}

The incidence of ILD-LC should not be ignored, and be more aware of the development of this disease and follow-up of ILD patients in clinical practice. Before lung transplantation in ILD end-stage patients, a variety of methods are needed to find the hidden LC. This study reveals that ILD combined LC differs from LC in clinical manifestations, pathological characteristics 
A
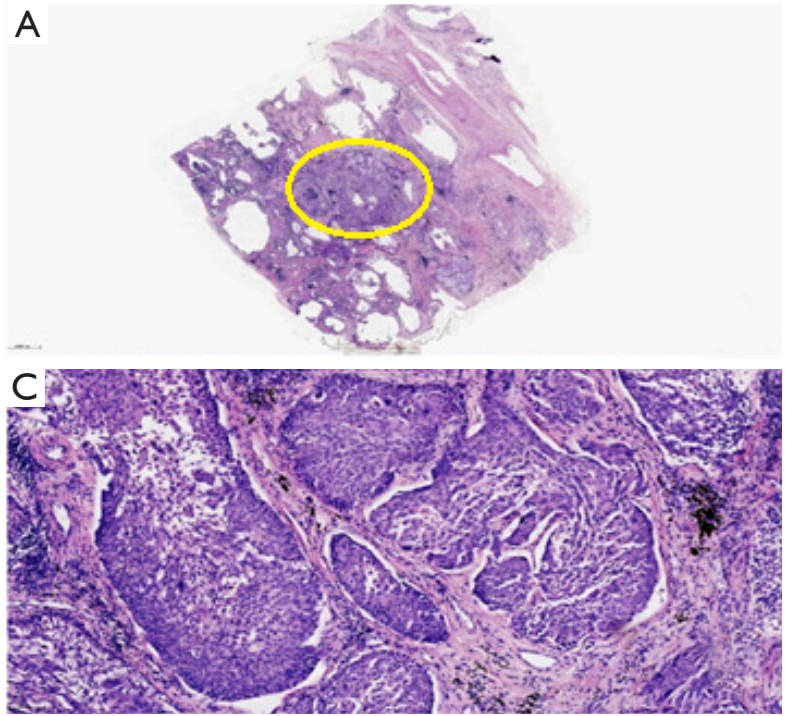

E
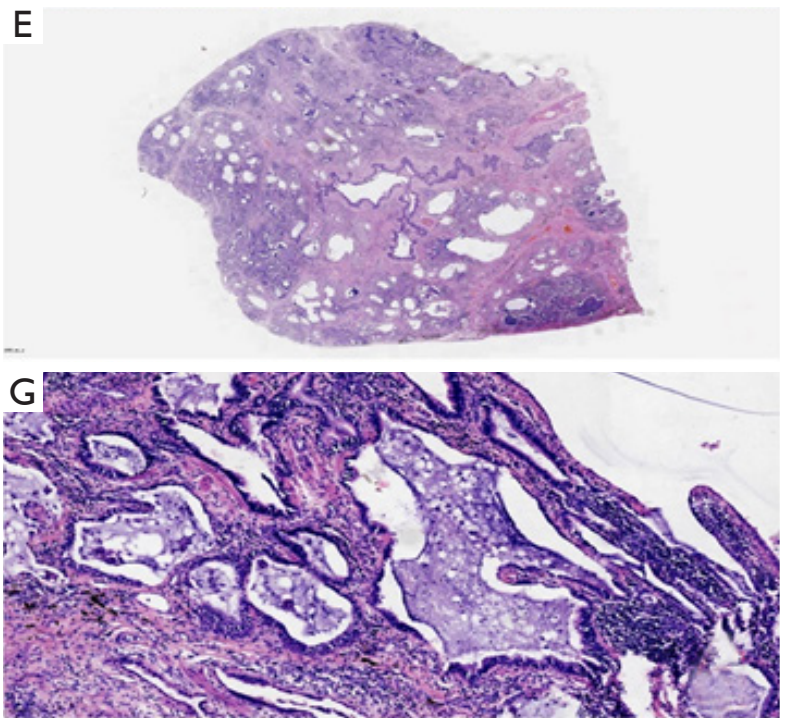
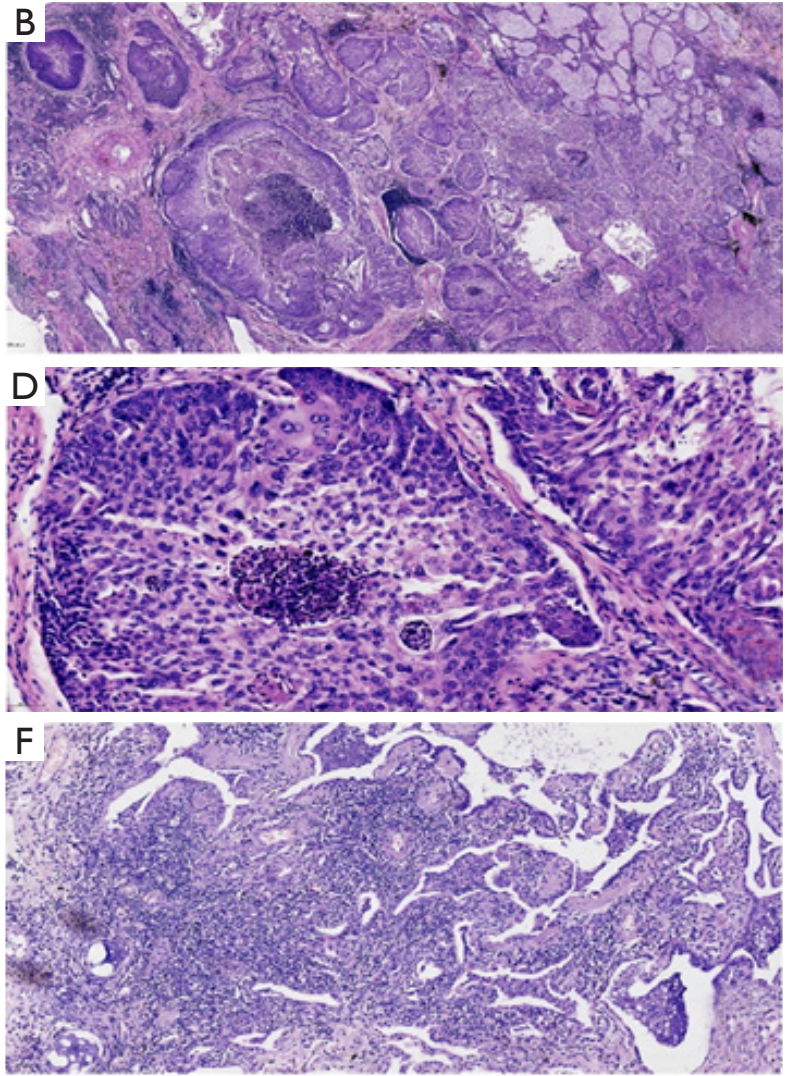

$\mathrm{H}$

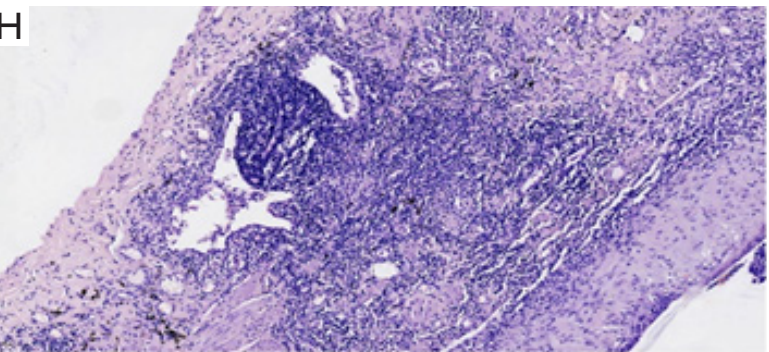

Figure $3 \mathrm{HE}$ staining of lung tissue in No. 4 patients. (A) HE staining of the tumor site in the pulmonary parenchyma near the hilum of the lung, ultra-low power scanning section (yellow circle). The size is about $0.7 \mathrm{~cm}$, and the boundary for the surrounding lung tissue is apparent. (B) HE staining map of tumor cell mass, $\times 5$ times. The tumor showed infiltration growth, the size of the nests was different, and necrosis could be seen in the center of some nests. (C) HE staining map of tumor cell mass, $\times 20$ times. It showed invasive growth, cancerous interstitial hyperplasia, carbon deposition in the stroma, accompanied by lymphocyte infiltration and aggregation. (D) HE staining map of tumor cell mass, $\times 40$ times. The heteromorphism of tumor cells was obvious, keratosis could be seen in the nest, and tumor necrosis was found in the center of the nest. (E) HE staining of ultra-low power scanning sections. The structure of lung tissue was destroyed, interstitial fibrous tissue proliferated and honeycomb formed, $\times 1$ time. (F) HE staining of fibrous tissue, $\times 20$ times. There are a large number of lymphocytes infiltration and aggregation in the stroma of fibrous tissue hyperplasia. (G) HE staining of alveolar tissue, $\times 20$ times. The honeycomb structure was formed, a large number of inflammatory cells infiltrated in the stroma, the cyst cavity was covered with ciliated columnar epithelium, and the mucus and inflammatory exudation and retention in the cavity. $(\mathrm{H})$ Pleural HE staining, $\times 20$ times. Pleural thickening, a large number of inflammatory cells infiltration and aggregation. 

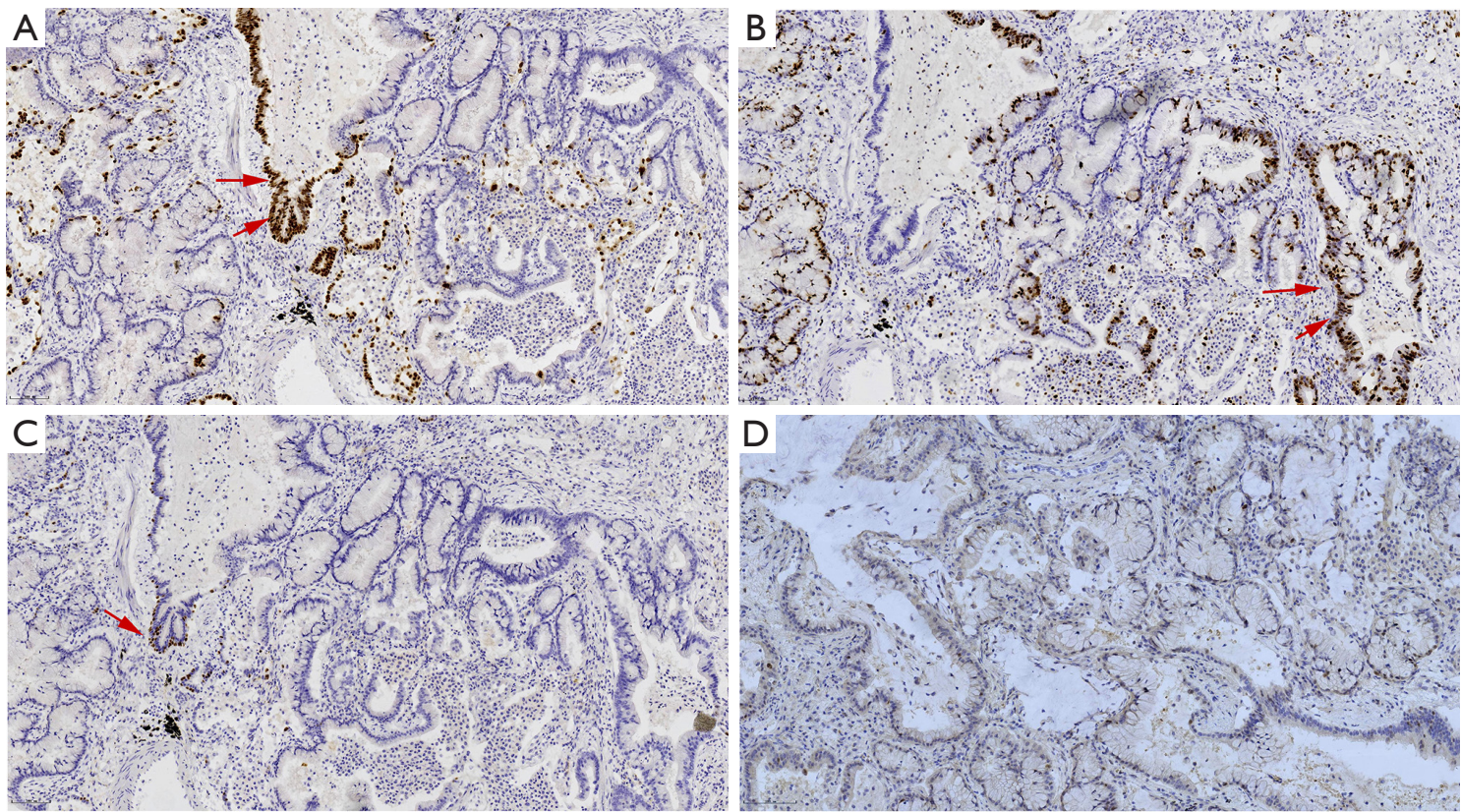

Figure 4 The immunohistochemical staining of lung tissue in No. 5 patients. (A) Immunohistochemical staining of TTF-1, $\times 20$ times. The bronchiole epithelium was TTF1 positive (shown by red arrow). Most of the other adenocarcinoma cells did not express TTF1, and only a few tumor cells were positive. (B) Immunohistochemical staining of Ki-67, $\times 20$ times. The positive expression of Ki-67 was found in the nucleus of the tumor, and about $50 \%$ of the tumor cells were positive. In contrast to neoplastic glands, the residual metaplastic mucous epithelium was Ki-67 negative (shown by the red arrow). (C) Immunohistochemical staining of p63, ×20 times. The basal nuclei were positive and the neoplastic glands were negative. The highly abnormal glands grew infiltratively and the basal cells disappeared. Metaplastic mucous epithelium of residual basal cells can be seen in the local lesions (shown by the red arrow). (D) Immunohistochemical staining of P53, ×20 times. Most tumor cells do not express P53.

Table 4 The follow-up of 7 patients with LC-CES-ILD

\begin{tabular}{|c|c|c|c|c|c|c|c|}
\hline Item & \multicolumn{7}{|c|}{ Case number (No.) } \\
\hline Follow-up (days) & 68 & 9 & 90 & 54 & 182 & 300 & 347 \\
\hline \multicolumn{8}{|l|}{ Follow-up results } \\
\hline No recurrence of tumor & + & & + & & & & \\
\hline Death & & + & & + & & & \\
\hline Causes of death & & Infected & & Infected & & & \\
\hline
\end{tabular}

and molecular phenotypes, and this finding has a guiding effect on future clinical practice.

\section{Acknowledgments}

Funding: This work was supported by the Talent
Introduction Project of China-Japan Friendship Hospital (Grant No. 2019-RC-1).

\section{Footnote}

Conflicts of Interest: All authors have completed the ICMJE 
uniform disclosure form (available at http://dx.doi. org/10.21037/tcr.2019.11.36). The authors have no conflicts of interest to declare.

Ethical Statement: The authors are accountable for all aspects of the work in ensuring that questions related to the accuracy or integrity of any part of the work are appropriately investigated and resolved. The study was conducted in accordance with the Declaration of Helsinki (as revised in 2013). This work was approved by the ethics committee at China-Japan Friendship Hospital (No. 2012$60)$. Informed consent was waived due to the retrospective nature of the study.

Open Access Statement: This is an Open Access article distributed in accordance with the Creative Commons Attribution-NonCommercial-NoDerivs 4.0 International License (CC BY-NC-ND 4.0), which permits the noncommercial replication and distribution of the article with the strict proviso that no changes or edits are made and the original work is properly cited (including links to both the formal publication through the relevant DOI and the license). See: https://creativecommons.org/licenses/by-nc-nd/4.0/.

\section{References}

1. American Thoracic Society; European Respiratory Society. American Thoracic Society/European Respiratory Society international multidisciplinary consensus classification of the idiopathic interstitial pneumonias. This joint statement of the American Thoracic Society (ATS), and the European Respiratory Society (ERS) was adopted by the ATS board of directors, June 2001 and by the ERS executive committee, June 2001. Am J Respir Crit Care Med 2002;165:277-304.

2. Weill D, Benden C, Corris PA, et al. A consensus document for the selection of lung transplant candidates: 2014 - an update from the Pulmonary Transplantation Council of the International Society for Heart and Lung Transplantation. J Heart Lung Transplant 2015;34:1-15.

3. Sheikine Y, Kuo FC, Lindeman NI. Clinical and Technical Aspects of Genomic Diagnostics for Precision Oncology. J Clin Oncol 2017;35:929-33.

4. Wallis A, Spinks KJB. The diagnosis and management of interstitial lung diseases. BMJ 2015;350:h2072.

5. Naccache JM, Gibiot Q, Monnet I, et al. Lung cancer and interstitial lung disease: a literature review. J Thorac Dis 2018;10:3829-44.

6. Tzouvelekis A, Gomatou G, Bouros E, et al. Common pathogenic mechanisms between idiopathic pulmonary fibrosis and lung cancer. Chest 2019;156:383-91.

7. Sucre JMS, Deutsch GH, Jetter CS, et al. A Shared Pattern of beta-Catenin Activation in Bronchopulmonary Dysplasia and Idiopathic Pulmonary Fibrosis. Am J Pathol 2018;188:853-62.

8. Choi WI, Lee DY, Choi HG, et al. Lung Cancer development and mortality in interstitial lung disease with and without connective tissue diseases: a five-year Nationwide population-based study. Respir Res 2019;20:117.

9. Yoo H, Jeong BH, Chung MJ, et al. Risk factors and clinical characteristics of lung cancer in idiopathic pulmonary fibrosis: a retrospective cohort study. BMC Pulm Med 2019;19:149.

10. Girard N, Marchand-Adam S, Naccache JM, et al. Lung cancer in combined pulmonary fibrosis and emphysema: a series of 47 Western patients. J Thorac Oncol 2014;9:1162-70.

11. Chatron E, Dégot T, Salvaterra E, et al. Lung cancer after lung transplantation: An analysis of 25 years of experience in a single institution. Clin Transplant 2019;33:e13446.

12. Ahmad U, Hakim AH, Tang A, et al. Patterns of Recurrence and Overall Survival in Incidental Lung Cancer in Explanted Lungs. Ann Thorac Surg 2019;107:891-896.

13. Hata A, Suzuki H, Nakajima T, et al. Concomitant Interstitial Lung Disease Is a Risk Factor for Pleural Invasion in Lung Cancer. Ann Thorac Surg 2017;103:967-74.

14. Zhu WY, Hu XF, Fang KX, et al. Prognostic value of mutant p53, Ki-67, and TTF-1 and their correlation with EGFR mutation in patients with non-small cell lung cancer. Histol Histopathol 2019;34:1269-78.

15. Folescu R, Levai CM, Grigoras ML, et al. Expression and significance of Ki-67 in lung cancer. Rom J Morphol Embryol 2018;59:227-33.

16. Fujimoto D, Tomii K, Otoshi T, et al. Preexisting interstitial lung disease is inversely correlated to tumor epidermal growth factor receptor mutation in patients with lung adenocarcinoma. Lung Cancer 2013;80:159-64.

17. Masai K, Tsuta K, Motoi N, et al. Clinicopathological, Immunohistochemical, and Genetic Features of Primary Lung Adenocarcinoma Occurring in the Setting of Usual Interstitial Pneumonia Pattern. J Thorac Oncol 2016;11:2141-9.

Cite this article as: Wang B, Zhang $\mathrm{X}$, Chen H, Yang L, Li J, Xiao F, Liang C, Zhong D. The clinicopathological study of lung cancer concealed in end-stage of interstitial lung disease. Transl Cancer Res 2020;9(2):536-546. doi: 10.21037/ tcr.2019.11.36 
Supplementary

Table S1 Gene locus information in this study

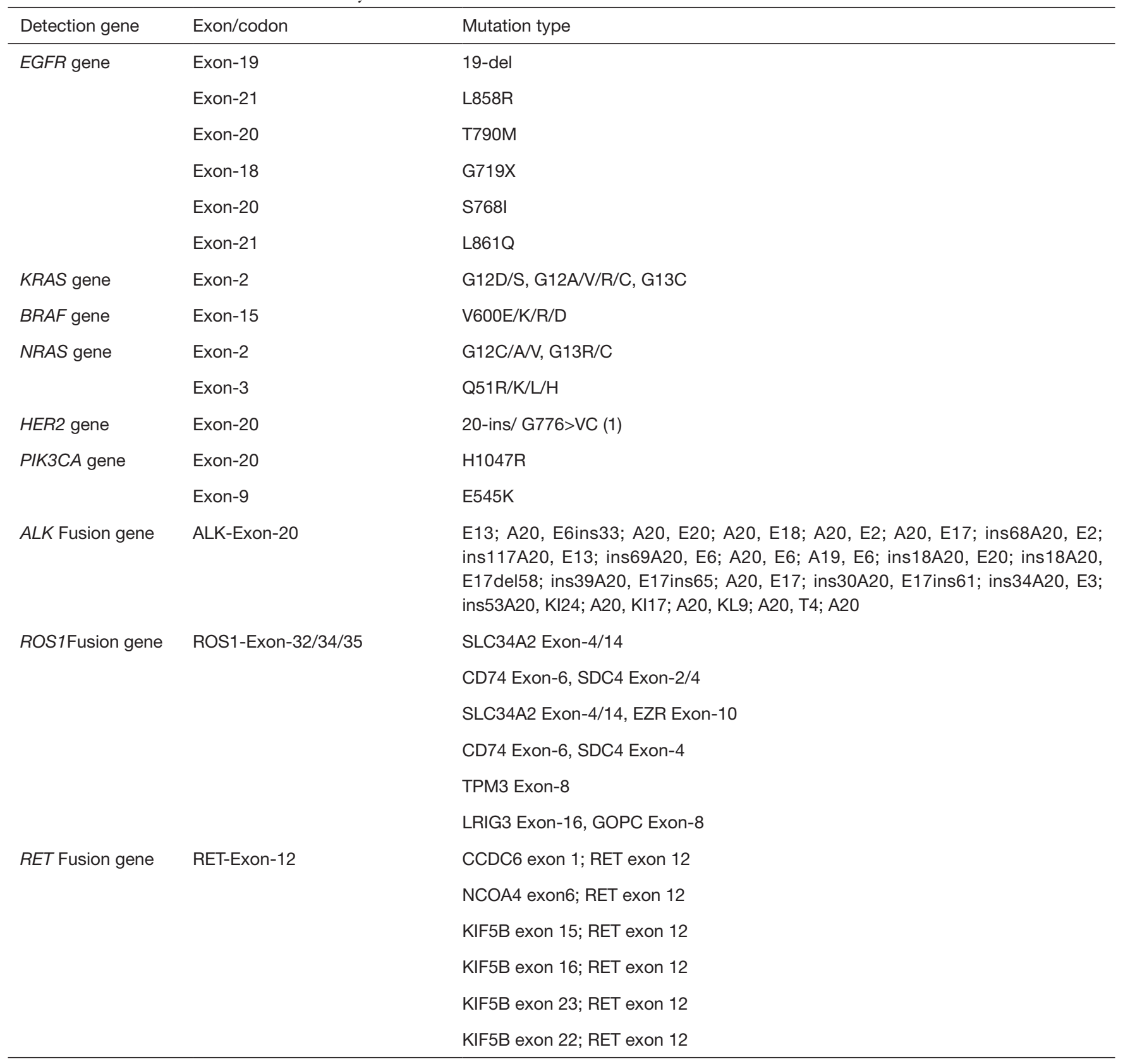


Table S2 The results of molecular pathological examination

\begin{tabular}{|c|c|c|c|c|c|c|c|}
\hline Item & \multicolumn{7}{|c|}{ Case number } \\
\hline NRAS & - & - & - & - & - & - & - \\
\hline EGFR & - & - & - & - & - & - & - \\
\hline$A L K$ & - & - & - & - & - & - & - \\
\hline Braf & - & - & - & - & - & - & - \\
\hline Her-2 & - & - & - & - & - & - & - \\
\hline РІЗКСА & - & - & - & - & - & - & - \\
\hline$R E T$ & - & - & - & - & - & - & - \\
\hline
\end{tabular}

- means that the results of examination are negative and + means that the results of examination are positive. The test results in $K R A S$ in No. 6 patients were exon2 G12A 35G>C, exon2 G12V 35G>T, exon2 G12R 34G>C, exon2 G12C 34G>T, exon2 G13C 37G>T. 